\title{
Galactic neutral hydrogen and the magnetic ISM foreground
}

\author{
S.E. Clark ${ }^{1,2}$ \\ ${ }^{1}$ Institute for Advanced Study, \\ 1 Einstein Drive, Princeton, New Jersey, USA \\ email: seclark@ias.edu \\ ${ }^{2}$ Hubble Fellow
}

\begin{abstract}
The interstellar medium is suffused with magnetic fields, which inform the shape of structures in the diffuse gas. Recent high-dynamic range observations of Galactic neutral hydrogen, combined with novel data analysis techniques, have revealed a deep link between the morphology of neutral gas and the ambient magnetic field. At the same time, an observational revolution is underway in low-frequency radio polarimetry, driven in part by the need to characterize foregrounds to the cosmological 21-cm signal. A new generation of experiments, capable of high angular and Faraday depth resolution, are revealing complex filamentary structures in diffuse polarization. The relationship between filamentary structures observed in radio-polarimetric data and those observed in atomic hydrogen is not yet well understood. Multiwavelength observations will enable new insights into the magnetic interstellar medium across phases.
\end{abstract}

Keywords. magnetic fields, polarization, ISM: structure, ISM: magnetic fields, cosmology: observations

\section{Introduction}

Detection of the redshifted 21-cm signal from the Epoch of Reionization (EoR) is a formidable challenge because Galactic foreground emission dominates at EoR frequencies ( $\sim 100-200 \mathrm{MHz}$, corresponding to 21-cm emission at a redshift of $z \sim 6-12$ ). The Galactic emission at these frequencies is primarily diffuse synchrotron emission from cosmic rays accelerated in the Galactic magnetic field. The partially polarized synchrotron emission is Faraday rotated - that is, its plane of polarization is rotated as it traverses the magneto-ionic medium in the Galaxy. Foreground polarization structures can leak into measurements of the total intensity and mimic the cosmological 21-cm signal, so this diffuse foreground polarization must be well-characterized (see, e.g., Jelić et al. 2010). Thus, the search for the EoR signal is driving new observations of the Galactic magnetoionic medium: an opportunity for synergy between EoR science and the study of the interstellar medium (ISM).

Independent efforts have characterized the diffuse Galactic neutral medium with unprecedented sensitivity. Several large-area surveys of the Galactic $(z=0) 21$-cm sky have recently been completed, including the Galactic Arecibo L-Band Feed Array Survey (GALFA-Hi; Peek et al. 2011, Peek et al. 2017), which mapped 13,000 deg ${ }^{2}$ with $F W H M \sim 4^{\prime}$ angular resolution and $0.18 \mathrm{~km} \mathrm{~s}^{-1}$ spectral resolution. An all-sky Hi survey at lower resolution ( $\left.\sim 16^{\prime}\right)$, the Hi $4 \pi$ Survey (HI4PI; Bekhti et al. 2016), was produced by combining the $\sim 11^{\prime}$ Effelsberg-Bonn Hi Survey (EBHIS; Winkel et al. 2016) of the northern Celestial sky with the 16' Galactic All-Sky Survey (GASS) carried out with the Parkes telescope. Together, these surveys enable detailed study of the diffuse neutral medium over large areas of sky. 


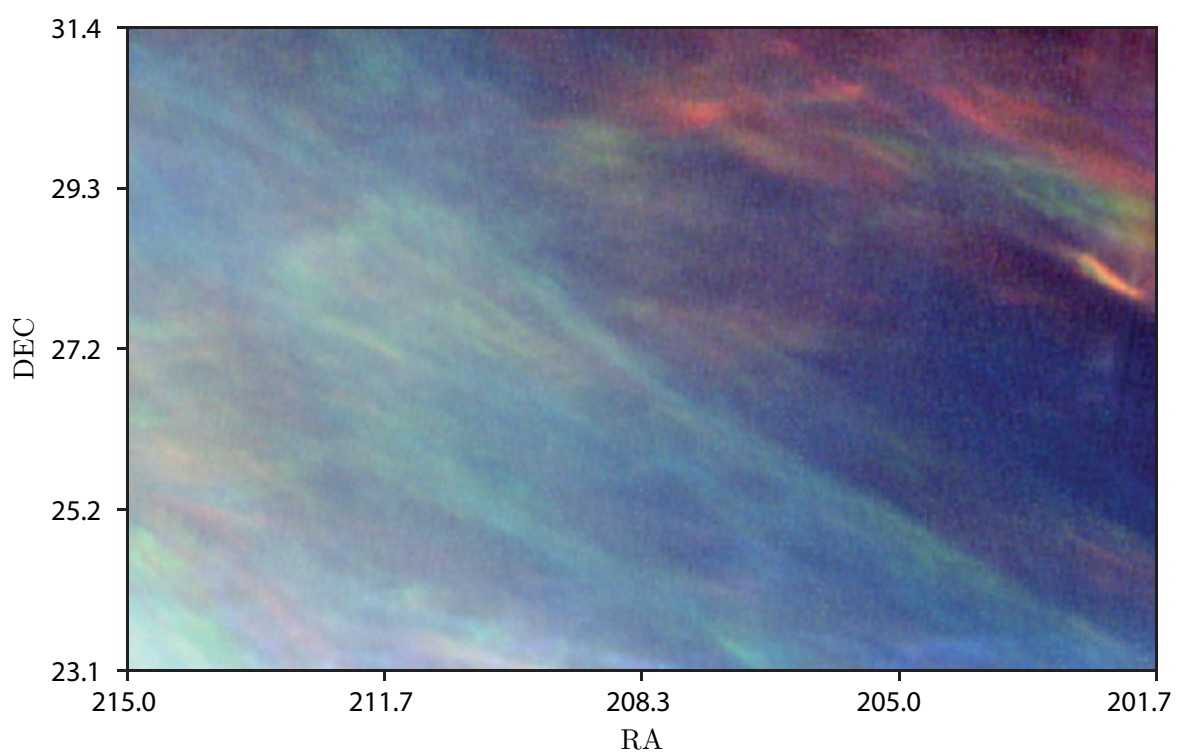

Figure 1. A representative section of diffuse neutral hydrogen in the high Galactic latitude GALFA-Hi sky. The data is from GALFA-Hi Data Release 2 (Peek et al. 2017). The image is colored by line-of-sight velocity: the red, green, and blue image components correspond to the integrated $\mathrm{HI}$ brightness in three velocity channels: $-7 \mathrm{~km} \mathrm{~s}^{-1}$ to $-4 \mathrm{~km} \mathrm{~s}^{-1},-3.3 \mathrm{~km}$ $\mathrm{s}^{-1}$ to $-0.4 \mathrm{~km} \mathrm{~s}^{-1}$, and $0.4 \mathrm{~km} \mathrm{~s}^{-1}$ to $3.3 \mathrm{~km} \mathrm{~s}^{-1}$, respectively. Each channel is displayed in a logarithmic stretch, with minimum and maximum values mapped to RGB image values of 0 and 1 , respectively. The Hi data in these thin velocity channels is highly linear, characterized by high aspect ratio features. These are the Hi fibers described in Clark et al. (2014) and found to be aligned with the plane-of-sky magnetic field orientation.

\section{Neutral hydrogen and the magnetic field}

The high dynamic range of the GALFA-Hi survey, in combination with a novel machine vision algorithm, led to the discovery that the cold, diffuse Hi that covers the high Galactic latitude sky is organized into thin, linear filamentary features, or "Hi fibers", that are extremely well aligned with the ambient magnetic field (Clark et al. 2014). Clark et al. (2014) developed the Rolling Hough Transform (RHT), an algorithm that measures the coherent linearity of image features as a function of orientation. The local orientation of linear features in GALFA-Hi channel maps was shown to be in striking agreement with the local orientation of the plane-of-sky magnetic field, as traced by optical starlight polarization (Clark et al. 2014) and by polarized thermal dust emission at $353 \mathrm{GHz}$ (Clark et al. 2015).

The Hi fibers are most apparent - and their alignment with the magnetic field is most pronounced - in high-resolution observations, but filamentary structures have also been characterized in the EBHIS and GASS data sets (Kalberla et al. 2016). These analyses broadly agree that the diffuse cold neutral medium is generically described by diffuse $\left(N_{H} \sim 10^{19} \mathrm{~cm}^{-2}\right)$ filamentary structures that align with the plane-of-sky magnetic field orientation. Because the structures are viewed in projection, their three-dimensional structure is not known, and they have been interpreted both as "filaments" - i.e, as having an approximately cylindrical geometry - and as "sheets" viewed edge-on (see discussion in Kalberla et al. 2016).

In addition to tracing the plane-of-sky magnetic field orientation, recent work has shown that the velocity structure of Hi morphology can be used to trace the degree 
to which the magnetic field is tangled along the line of sight (Clark 2017, in prep). Linear structures in Hi are most prominent in thin velocity channels, and Clark (2017) shows that regions of sky where the orientation of these Hi structures is coherent as a function of line-of-sight velocity correspond to areas of high fractional polarization in $353 \mathrm{GHz}$ polarized thermal dust emission. Likewise, regions of sky where Hi regions are detected at multiple orientations correspond to lower fractional dust polarization. This is consistent with a picture in which Hi structures trace the local magnetic field orientation, and multiply oriented magnetic fields along a given line of sight depolarize the dust emission. Comparison of these data to diffuse synchrotron emission may be used to map the magnetic field along the line of sight.

At high Galactic latitudes, the bulk of the dust, and therefore of the neutral gas, lies in the Local Bubble wall, the edge of an evacuated cavity that extends to a distance of roughly $100 \mathrm{pc}$ from the Sun (Lallement et al. 2003). Clark et al. (2014) found that the magnetically aligned Hi fibers are most likely a component of the wall of the Local Bubble. The magnetically aligned structures in the Local Bubble may be related to the Bubble's formation - that is, to supernova explosions that blew a bubble into the local ISM, stretching and inflating the magnetic field lines (Cox \& Reynolds 1987).

\section{Neutral hydrogen and the magneto-ionic medium}

Recent low frequency radio polarimetry experiments have mapped small regions of the interstellar medium with high angular and Faraday depth resolution. Experiments such as the Low Frequency Array (LOFAR) and the Murchison Widefield Array (MWA) have observed rich morphological structure, including high aspect ratio filamentary structures (Jelić et al. 2014, Zaroubi et al. 2015, Lenc et al. 2016). One particularly well-studied region is the 3C196 LOFAR field (Jelić et al. 2015, Zaroubi et al. 2015). This region contains strikingly straight Faraday structures that, at least for the most prominent linear features, are aligned with the Planck $353 \mathrm{GHz}$ magnetic field. It seems reasonable that a common physical property influences both the field structure and the morphology of the Faraday features, but the physical conditions that give rise to the Faraday structures are not well understood. In addition, other LOFAR fields with similarly linear Faraday structures do not show the same alignment with the magnetic field traced by polarized dust emission (Zaroubi et al. 2015). The dust polarization traces the density-weighted plane-of-sky magnetic field orientation, and this line-of-sight averaging may in general obscure any localized correlations.

At least in 3C196, the association between the orientation of linear structure and the plane-of-sky magnetic field orientation is reminiscent of the Clark et al. (2014) HI features. Are there diffuse Hi features associated with these Faraday structures? 3C196 is unfortunately outside of the GALFA-Hi observable region, but is covered by the EBHIS survey. Kalberla \& Kerp (2016) found filamentary Hi structure aligned in the same orientation as both the Faraday depth structures and the Planck-measured magnetic field (see also Kalberla et al. 2017).

Through multiwavelength studies, a clearer picture of the three-dimensional relationship between the neutral sky and the Faraday sky is beginning to emerge. Van Eck et al. (2017) analyzed a LOFAR region that shows rich polarized structure, including extremely thin, straight depolarization canals. The authors use a three-dimensional dust map constructed from optical reddening measurements (Green et al. 2015) to identify two neutral clouds along the line of sight in the LOFAR field. Using the estimated sizes and distances of these neutral clouds, the authors model the observed emission via a picture in which the bulk of the Faraday thin emission originates in the neutral clouds, with 
interleaving ionized regions Faraday rotating the signal. The success of this method in explaining the observations is tantalizing, because if Faraday observations can be reliably mapped to distance-resolved neutral structures, this will be a powerful method for mapping the component of the magnetic field parallel to the line of sight in the Galaxy. This technique is complimentary to starlight polarization measurements, which, in combination with precise stellar distance measurements, can constrain the plane-of-sky magnetic field orientation as a function of distance.

\section{Future prospects}

As the neutral and magneto-ionic components of the diffuse ISM have been better resolved by observations, new and mysterious structures have been found. Thin, linear Hi structures abound in the high Galactic latitude sky, and are aligned with the planeof-sky magnetic field traced by magnetically aligned interstellar dust grains (Clark et al. 2014, Clark et al. 2015). Thin, linear Faraday depth structures are similarly ubiquitous, at least in the few regions of sky that have been mapped with sufficient angular and Faraday depth resolution (Jelić et al. 2014, Jelić et al. 2015, Lenc et al. 2016). These distinct tracers, of the cold neutral medium and the magneto-ionic medium, may be key to understanding the nature of the Galactic magnetic field within and between phases of the ISM. Multiwavelength analyses and novel data sets, driven in part by a need to understand EoR foregrounds, will enable a better understanding of the magnetic, multiphase ISM.

\section{Acknowledgements}

S.E.C. is supported by NASA through Hubble Fellowship grant \#HST-HF2-51389.001A awarded by the Space Telescope Science Institute, which is operated by the Association of Universities for Research in Astronomy, Inc., for NASA, under contract NAS5-26555. This publication utilizes data from Galactic ALFA HI (GALFA-HI) survey data set obtained with the Arecibo L-band Feed Array (ALFA) on the Arecibo 305m telescope. The Arecibo Observatory is operated by SRI International under a cooperative agreement with the National Science Foundation (AST-1100968), and in alliance with Ana G. Méndez-Universidad Metropolitana, and the Universities Space Research Association. The GALFA-HI surveys have been funded by the NSF through grants to Columbia University, the University of Wisconsin, and the University of California.

\section{References}

Bekhti, B. N., Flöer, L., Keller, R., Kerp, J., Lenz, D., Winkel, B., Bailin, J., Calabretta, M. R., Dedes, L., Ford, H. A., Gibson, B. K., Haud, U., Janowiecki, S., Kalberla, P. M. W., Lockman, F. J., McClure-Griffiths, N. M., Murphy, T., Nakanishi, H., Pisano, D. J., \& Staveley-Smith, L. A\&BA, 594, 116

Clark, S. E., Peek, J. E. G., \& Putman, M. E. 2014, ApJ, 789, 82

Clark, S. E., Hill, J. C., Peek, J. E. G., Putman, M. E., \& Babler, B. 2015, Phys. Rev. Lett.,115, 241302

Clark, S. E., 2018, arXiv:1802.00011

Cox, D. P. \& Reynolds, R. J., 1987, A\& ARv, 25, 303

Green, G. M., Schlafly, E. F., Finkbeiner, D. P., Rix, H.-W., Martin, N., Burgett, W., Draper, P. W., Flewelling, H., Hodapp, K., Kaiser, N., Kudritzki, R. P., Magnier, E., Metcalfe, N., Price, P., Tonry, J., \& Wainscoat, R., 2015, ApJ, 810, 25

Jelić, V., Zaroubi, S., Labropoulos, P., Bernardi, G., de Bruyn, A. G., \& Koopmans, L.V. E., 2010 MNRAS, 409, 1647 
Jelić, V., de Bruyn, A. G., Mevius, M., Abdalla, F. B., Asad, K. M. B., Bernardi, G., Brentjens, M. A., Bus, S., Chapman, E., Ciardi, B., Daiboo, S., Fernandez, E. R., Ghosh, A., Harker, G., Jensen, H., Kazemi, S., Koopmans, L. V. E., Labropoulos, P., Martinez-Rubi, O., Mellema, G., Offringa, A. R., Pandey, V. N., Patil, A. H., Thomas, R. M., Vedantham, H. K., Veligatla, V., Yatawatta, S., Zaroubi, S., et al., 2014 A $8 A$, 568, 101

Jelić, V., de Bruyn, A. G., Pandey, V. N., et al. 2015, A\&A, 583, A137

Kalberla, P. M. W. \& Kerp, J., 2016, A\&\&A, 595, 37

Kalberla, P. M. W., Kerp, J., Haud, U., Winkel, B., Ben Bekhti, N., Flöer, L., \& Lenz, D. 2016, ApJ, 821, 2

Kalberla, P. M. W., Kerp, J., Haud, U., \& Haverkorn, M., 2017, A\& $A, 607,15$

Lallement, R., Welsh, B. Y., Vergely, J. L., Crifo, F., \& Sfeir, D., 2003, A\&̊A, 411, 447

Lenc, E., Gaensler, B. M., Sun, X. H., Sadler, E. M., Willis, A. G., Barry, N., Beardsley, A. P., Bell, M. E., Bernardi, G., Bowman, J. D., Briggs, F., Callingham, J. R., Cappallo, R. J., Carroll, P., Corey, B. E., de Oliveira-Costa, A., Deshpande, A. A., Dillon, J. S., Dwarkanath, K. S., Emrich, D., Ewall-Wice, A., Feng, L., For, B.-Q., Goeke, R., Greenhill, L. J., Hancock, P., Hazelton, B. J., Hewitt, J. N., Hindson, L., Hurley-Walker, N., JohnstonHollitt, M., et al., 2016, ApJ, 830, 38

Peek, J. E. G., Heiles, C., Douglas, K. A., Lee, M.-Y., Grcevich, J., Stanimirović, S., Putman, M. E., Korpela, E. J., Gibson, S. J., Begum, A., Saul, D., Robishaw, T., \& Krćo, M., 2011, ApJS, 194, 20

Peek, J. E. G., Babler, B. L., Zheng, Y. Zheng, Clark, S. E., Douglas, K. A., Korpela, E. J., Putman, M. E., Stanimirović, S., Gibson, S. J., \& Heiles., C., 2017, ApJS, accepted

Van Eck, C. L., Haverkorn, M., Alves, M. I. R., Beck, R., de Bruyn, A. G., Enßlin, T., Farnes, J. S., Ferrière, K., Heald, G., Horellou, C., Horneffer, A., Iacobelli, M., Jelić, V., MartíVidal, I., Mulcahy, D. D., Reich, W., Röttgering, H. J. A., Scaife, A. M. M., Schnitzeler, D. H. F. M., Sobey, C., \& Sridhar, S. S. 2017, A\& A, 597, 98

Winkel, B., Kerp, J., Flöer, L., Kalberla, P. M. W., Ben Bekhti, N., Keller, R., \& Lenz, D. 2016, $A \mathscr{G} A, 585,41$

Zaroubi, S., Jelić, V., de Bruyn, A. G., de Bruyn, A. G., Boulanger, F., Bracco, A., Kooistra, R., Alves, M. I. R., Brentjens, M. A., Ferrière, K., Ghosh, T., Koopmans, L. V. E., Levrier, F., Miville-Deschênes, M.-A., Montier, L., Pandey, V. N., \& Soler, J. D., 2015, MNRAS Letters, 454, L46 